\title{
CARBOHYDRATE AND LIGNIN CONTENTS IN PERENNIAL RYEGRASS (LOLIUM PERENNE L.) TREATED WITH SEA BAMBOO (ECKLONIA MAXIMA) EXTRACT AGAINST THE BACKGROUND OF NITROGEN FERTILISATION REGIME
}

\author{
GodlewsKa, A.* - CIEPIELA, G. A. \\ Siedlce University of Natural Sciences and Humanities, B. Prusa 14 st., 08-110 Siedlce, Poland \\ *Corresponding author \\ e-mail: agnieszka.godlewska@uph.edu.pl \\ (Received $16^{\text {th }}$ Mar 2020; accepted $2^{\text {nd }}$ Jul 2020)
}

\begin{abstract}
The objective of this study was to determine the effect of Ecklonia maxima extract on monosaccharide, structural and non-structural carbohydrate as well as lignin contents in two Lolium perenne cultivars against the background of a varied nitrogen fertilisation regime. A field experiment was arranged as a randomised sub-block design (split-split-plot) in Poland. The following factors were examined: biostimulant Kelpak SL applied at $2 \mathrm{dm}^{3} \cdot \mathrm{ha}^{-1}$ and a control (no biostimulant addition); nitrogen application rates of: $0,50,100$ and $150 \mathrm{~kg} \mathrm{ha}^{-1}$; cultivars of Lolium perenne: Diament and Gagat. The research reported here demonstrated positive effect of Ecklonia maxima extract on Lolium perenne chemical composition. Its results showed that structural carbohydrate and lignin contents in grass biomass may be reduced whereas non-structural carbohydrate content may be increased by means of an application of sea alga extract, which results in an improved quality of feed produced from these grasses. The biostimulant applied in combination with each nitrogen rate significantly increased the concentration of monosaccharides and non-structural carbohydrates, and reduced the concentration of structural carbohydrates and lignin in Lolium perenne biomass. Increasing nitrogen rates significantly increased cellulose, hemicellulose and lignin contents but they reduced amounts of monosaccharides and non-structural carbohydrates in the test grasses.
\end{abstract}

Keywords: Kelpak, biostimulant, fertilisation, grass, monosaccharides, cellulose, hemicellulose, non-structural carbohydrates

\section{Introduction}

As the reliance of agriculture's on chemicals (mineral fertilisers, plant protection agents) may negatively affect both food quality and the natural environment, innovative solutions have been increasingly sought (Niewiadomska et al., 2020). Due to this, in order to reduce such reliance, an alternative solution in the form of natural preparations, which include biostimulants, used as fertilisers is gaining more and more popularity all over the world (Colla and Rouphael, 2015; Sosnowski et al., 2016; Kocira et al., 2018a). An application of natural growth regulators may become one of components of modern agricultural practice. Scientific literature claims that these preparations are environmentally friendly, beneficially affect yield quality and quantity (Godlewska and Ciepiela, 2016; Caruso et al., 2019), and frequently eliminate a large part of biotic and abiotic stress (Rathore et al., 2009; Haider et al., 2012; Sharma et al., 2014). Simultaneously, an application of biostimulants may prove to be economically viable (Rengasamy et al., 2015). Sea algae extracts (division of green, red and brown algae) are biostimulants which have become quite popular in crop plant production (Du Jardin, 2015). Ecklonia maxima, a brown alga, is harvested off the coast of South Africa and along the Atlantic coast of Africa. Large amounts of this seaweed are deposited on the seashore, which results in environmental pollution. When collected, the organic matter 
may be utilised to produce the preparation called Kelpak which, in addition to plant hormones (cytokinins $(0.03 \mathrm{mg} / \mathrm{l})$ and auxins $(11 \mathrm{mg} / \mathrm{l})$, gibberellins, abscisic acid, brassinosteroids), contains polysaccharides (absent in terrestrial plants), amino acids, polyphenols as well as macro- and microelements (Strik et al., 2004; Papenfus et al., 2012; Rengasamy et al., 2015; Rauphael et al., 2017).

Although literature reports claim that sea algae extracts display a positive effect on many species of cultivated plants, the influencing process of those extracts have not be fully understood yet. The effect of biostimulants may, to a great extent, depend on the plant species and cultivar (Sultana et al., 2005; Battacharyya et al., 2015). There is still a paucity of information on an application of biostimulants to meadow plants.

The objective of this study was to determine the effect of Ecklonia maxima extract on monosaccharide, structural and non-structural carbohydrate as well as lignin contents in two Lolium perenne cultivars against the background of varied nitrogen fertilisation regime.

\section{Materials and Methods}

\section{Experimental Design}

A field experiment was arranged as a randomised sub-block design (split-split-plot) with three replicates at the Siedlce Experimental Unit of the University of Natural Sciences and Humanities in Poland $\left(52.169^{\circ} \mathrm{N}, 22.280^{\circ} \mathrm{E}\right)$ in late April, 2009. The plot area was $10 \mathrm{~m}^{2}$. The soil of the experimental site represents average soils, Hortic Anthrosol (WRB). Prior to the experiment set-up the characteristics of the soil were as follows: neutral $\mathrm{pH}$ ( $\mathrm{pH}$ in $1 \mathrm{n} \mathrm{KCl}=7.2)$, high humus content $(3.78 \%)$, high available phosphorus and magnesium contents $\left(\mathrm{P}_{2} \mathrm{O}_{5}-900 \mathrm{mg} \cdot \mathrm{kg}^{-1}, \mathrm{Mg}-84 \mathrm{mg} \cdot \mathrm{kg}^{-1}\right)$ and average total nitrogen and available potassium contents $\left(\mathrm{N}-1.8 \mathrm{~g} \cdot \mathrm{kg}^{-1} \mathrm{DM}, \mathrm{K}_{2} \mathrm{O}-190 \mathrm{mg} \mathrm{kg}^{-1}\right)$. Soil chemical analysis was carried out at an accredited laboratory of the Chemical and Agricultural Research Laboratory in Warsaw (Poland). Available phosphorus and potassium in the soil were extracted by means of the Egner-Riehm method (Staugaitis and Rutkauskiene, 2012) and available magnesium - using the Schachtschabel method (Staugaitis and Rutkauskiene, 2012). Phosphorus was determined by the colorimetric method, total nitrogen by the Kjeldahl method and potassium and magnesium by the atomic absorption spectrophotometry AAS. The following factors were examined:

- biostimulant (sea bamboo extract) with the trade name Kelpak SL applied at $2 \mathrm{dm}^{3} \cdot \mathrm{ha}^{-1}$ and a control (no biostimulant addition);

- nitrogen application rate: $0,50,100$ and $150 \mathrm{~kg} \mathrm{ha}^{-1}$;

- cultivars of Lolium perenne: Diament and Gagat.

The growth stimulant applied in the experiment is an extract from the fastest growing seaweed (kelp) Ecklonia maxima harvested off the coast of South Africa. The extract contains, among others, the natural plant hormones auxins $\left(11 \mathrm{mg} \mathrm{L}^{-1}\right.$ and cytokinins $\left(0.03 \mathrm{mg} . \mathrm{L}^{-1}\right)$. The commercial name of the stimulant is Kelpak SL, and it is manufactured by Kelp Products (Pty) Ltd. P.O. Box 325, Simon's Town, the Republic of South Africa.

The sowing amount of Lolium perenne L. variety Gagat and Dukat was calculated on the basis of standards developed by Research Centre for Cultivar Testing (COBORU, 2008). Standard cultivation methods were used in the experiment, and additionally the plants were fertilized with various doses of nitrogen and the Kalpak SL biostimulator. 
The grass sowing rate was $38 \mathrm{~kg} \mathrm{ha}^{-1}$ (TWG - thousand-grain weight - $3.2 \mathrm{~g}, 1187$ kernels per square meter).

In the growing season, when the experiment was set up, neither the nitrogen regime nor the biostimulant were applied. The season was an introductory period when three weed-control cuttings were made. After the second cutting, mineral fertilisation was applied to all the plots at the rates of $30 \mathrm{~kg}$ ha $\mathrm{N}$ (ammonium nitrate) and $30 \mathrm{~kg}$.ha $\mathrm{K}_{2} \mathrm{O}$ (potash salt). Phosphorus was not applied as the soil was rich in available forms of this element. Over the study period (2010-2012), the cutting regime was three harvests per year. Ammonium nitrate was applied three times per year. The total nitrogen amount was split into three equal rates which were applied before each cutting. Phosphorus and potassium needs of the grass were calculated taking into account the expected dry matter yields, the appropriate (from the ruminant nutrition standpoint) mineral contents of hay as well as soil $\mathrm{P}$ and $\mathrm{K}$ availability.

Phosphorus and potassium fertilisation was applied to all the plots. Phosphorus was applied once as triple superphosphate at a rate of $40 \mathrm{~kg} \mathrm{ha} \mathrm{P}_{2} \mathrm{O}_{5}$ in spring. The amount of potassium $\left(160 \mathrm{~kg}\right.$ ha $\left.\mathrm{K}_{2} \mathrm{O}\right)$ was split into three equal rates and applied prior to each cutting as $60 \%$ potash salt. The biostimulant was sprayed as an aqueous solution; the rate was $2 \mathrm{dm}^{3}$ of biostimulant per hectare diluted in water to $400 \mathrm{dm}^{3}$. The spraying the rate $2 \mathrm{dm}^{3}$ of biostimulant was performed before each cutting: the first application was three weeks before the first cutting, the second one two weeks after the first harvest and the last cutting three weeks after the second harvest.

\section{Weather Conditions}

Weather conditions differed during the study period (Table 1). Average air temperatures and precipitation sums in all the growing seasons were higher than the long-term means and the precipitation was very unevenly distributed. In 2010 and 2011 rainfall was, respectively, by 115.3 and $80.5 \mathrm{~mm}$ higher than the long-term means. It is worth noticing that in July 2011 the precipitation was 4.5 times higher than the long-term mean for July, and it constituted $48 \%$ rainfall of the whole growing season. Also in June 2012 precipitation was by $50.5 \mathrm{~mm}$ higher compared with the long-term mean for this month. By contrast, high rainfall shortages were recorded in April 2010, September 2011 and 2012.

Table 1. Meteorological condition in growing season 2010-2012 by meteorological station in Siedlce

\begin{tabular}{|c|c|c|c|c|c|c|c|}
\hline \multirow{2}{*}{ Years } & \multicolumn{6}{|c|}{ Means air temperatures $\left({ }^{0} \mathrm{C}\right)$} & \multirow{2}{*}{$\begin{array}{c}\text { Mean daily air } \\
\text { temperature in growing } \\
\text { season }\left({ }^{0} \mathrm{C}\right) \\
\end{array}$} \\
\hline & IV & $\mathrm{V}$ & VI & VII & VIII & IX & \\
\hline 2010 & 8.9 & 14.0 & 17.4 & 21.6 & 19.8 & 11.8 & 15.6 \\
\hline 2011 & 9.8 & 13.4 & 18.1 & 18.2 & 18.1 & 14.4 & 15.3 \\
\hline 2012 & 9.0 & 14.5 & 16.4 & 20.4 & 18.0 & 14.2 & 15.4 \\
\hline $\begin{array}{c}\text { Means of many years } \\
(2002-2012)\end{array}$ & 7.7 & 10.0 & 16.1 & 19.3 & 18.0 & 13.0 & 14.0 \\
\hline \multirow{2}{*}{ Years } & \multicolumn{6}{|c|}{ Monthly precipitations (mm) } & Sum of precipitation in \\
\hline & IV & $\mathrm{V}$ & VI & VII & VIII & IX & growing season $(\mathbf{m m})$ \\
\hline 2010 & 10.7 & 93.2 & 62.6 & 77.0 & 106.3 & 109.9 & 459.7 \\
\hline 2011 & 38.1 & 55.6 & 44.3 & 204.2 & 55.4 & 26.6 & 424.2 \\
\hline 2012 & 40.3 & 59.7 & 118.7 & 41.4 & 64.1 & 30.8 & 355.0 \\
\hline $\begin{array}{c}\text { Means of many years } \\
(2002-2012)\end{array}$ & 52.3 & 50.0 & 68.2 & 45.7 & 66.8 & 60.7 & 343.7 \\
\hline
\end{tabular}

Reported by the meteorological station in Siedlce 


\section{Chemical Analysis}

During harvest of each cut, green matter from each plot (whole area, that is $10 \mathrm{~m}^{2}$ ) was weighed to determine the yield, and $0.5 \mathrm{~kg}$ green matter samples of grass were taken to determine the drying-up coefficient and to carry out chemical analyses. The samples were left to dry in a ventilated room. Airy dry matter was weighed (to determine dry matter yield per plot) and was then shredded and ground. The obtained material was subjected to chemical analysis to determine dry matter, monosaccharides, cellulose, hemicellulose, lignin, total protein, crude ash and crude fat. The components were determined in powdered dry plant material placed on Petri dishes by near infrared spectroscopy (NIRS) using a NIRFlex N-500 spectrometer and ready-to-use INGOT ${ }^{\circledR}$ calibration applications. INGOT ${ }^{\circledR}$ is a set of Universal NIR calibrations (adapter to the NIRFlex N-500 data format) for the analysis of raw materials and finished products, e.g. grass. Non-structural carbohydrates were calculated following Virkajärvi et al. (2012):

$$
\begin{gathered}
\text { Non-structural carbohydrates }=1000-(\text { total protein }+ \text { crude ash }+ \\
\text { crude fat }+ \text { cellulose }+ \text { hemicelluloses }+ \text { lignin })
\end{gathered}
$$

\section{Statistical Analysis}

Means presented in tables 2-6 for each factor (biostimulant: $\mathrm{N}$ rate; cultivar; cut) and for interactions between these factors were calculated (for each characteristic studied) using all the data for individual experimental combinations obtained in replicates from each cut in all the study years. The data was processed by means of STATISTICA StatSoft, Inc. (2011) (data analysis software system), version 10 (www.statsoft.com). The program was used to conduct variance analysis (ANOVA/MANOVA). Significance of differences between means for the experimental factors was checked using Tukey's test at the significance level of $\alpha \leq 0.05$.

\section{Results and Discussion}

The concentration of monosaccharides, structural and non-structural carbohydrates as well as lignin is an important criterion of forage grass assessment. Monosaccharide content in both Lolium perenne cultivars (Table 2) depended on all the experimental factors. Regardless of the nitrogen fertilisation regime, cuts and cultivars, the sea alga extract significantly increased monosaccharide content in Lolium perenne biomass, which improved the forage quality. Kelpak application increased monosaccharide content by $31.8 \%$ in cv. Gagat biomass, and by $40.7 \%$ in cv. Diament biomass, which indicates that the effect of the biostimulants was cultivar-related. A positive effect of sea algae extracts on the concentration of the discussed compounds in the biomass of various plant species has been confirmed by Pacholczak et al. (2012), Sridhar and Rengasamy (2011) as well as El-Miniawy et al. (2014). The results of the study reported here indicated a significant interaction of the biostimulant with nitrogen rates. The highest increase in carbohydrate content following Ecklonia maxima extract application, amounting to as much as $44.3 \%$, was recorded in the biomass of grasses cultivated in the plots without nitrogen fertiliser. In earlier studies, Godlewska and Ciepiela (2013) demonstrated that the biostimulant Kelpak applied to units without nitrogen fertiliser contributed to an average increase of $42.4 \%$ in the sugar content in the biomass of orchard grass and Braun's festulolium. 
Table 2. Content of monosaccharides in Lolium perenne $\left(\mathrm{g} . \mathrm{kg}^{-1} \mathrm{~s} . \mathrm{m}\right.$.) depending on biostimulator, nitrogen dose and cut (mean from years 2010-2012)

\begin{tabular}{|c|c|c|c|c|c|c|c|c|c|c|}
\hline \multirow{4}{*}{ Cut } & \multirow{4}{*}{$\begin{array}{c}\text { N dose } \\
\left(\mathrm{kg} \mathrm{N}^{\bullet} \mathrm{ha}^{-1}\right)\end{array}$} & \multicolumn{4}{|c|}{ Cultivar } & \multirow{2}{*}{\multicolumn{2}{|c|}{ Mean }} & \multicolumn{2}{|c|}{ Cultivar } & \multirow{4}{*}{ Mean } \\
\hline & & Dia & ent & Ga & & & & Diament & Gagat & \\
\hline & & \multicolumn{4}{|c|}{ Dose of Klepak $\left(\mathbf{d m}^{3} \bullet \mathrm{ha}^{-1}\right)$} & \multicolumn{2}{|c|}{ Dose of Klepak } & \multirow{2}{*}{\multicolumn{2}{|c|}{ Mean }} & \\
\hline & & $\mathbf{0}$ & 2 & 0 & 2 & 0 & 2 & & & \\
\hline \multirow{4}{*}{1} & 0 & $117.6 \mathrm{a}$ & $174.9 \mathrm{~b}$ & $124.6 \mathrm{a}$ & $165.6 \mathrm{~b}$ & $121.1 \mathrm{a}$ & $170.2 \mathrm{~b}$ & $146.2 \mathrm{~A}$ & $145.1 \mathrm{~A}$ & $145.7 \mathrm{~A}$ \\
\hline & 50 & & $.9 \mathrm{~b}$ & $117.9 \mathrm{a}$ & $148.1 \mathrm{~b}$ & $113.2 \mathrm{a}$ & $151.5 \mathrm{~b}$ & $131.7 \mathrm{AB}$ & $133.0 \mathrm{AB}$ & $132.4 \mathrm{AB}$ \\
\hline & 100 & 101 & & $112.3 \mathrm{a}$ & & $107.1 \mathrm{a}$ & $135.6 \mathrm{~b}$ & $116.9 \mathrm{~B}$ & $125.8 \mathrm{AB}$ & $121.4 \mathrm{~B}$ \\
\hline & 150 & & & $107.7 \mathrm{a}$ & & & & $110.1 \mathrm{BC}$ & $116.1 \mathrm{~B}$ & $113.1 \mathrm{BC}$ \\
\hline \multirow{4}{*}{2} & 0 & & $0 \mathrm{~b}$ & $114.6 \mathrm{a}$ & & $106.7 \mathrm{a}$ & $1 \mathrm{~b}$ & $122.9 \mathrm{~A}$ & $138.9 \mathrm{~A}$ & $130.9 \mathrm{~A}$ \\
\hline & 50 & $95.6 \mathrm{a}$ & $132.8 \mathrm{~b}$ & $103.6 \mathrm{a}$ & $138.0 \mathrm{~b}$ & $99.6 \mathrm{a}$ & $135.4 \mathrm{~b}$ & $114.2 \mathrm{~A}$ & $120.8 \mathrm{~B}$ & $117.5 \mathrm{~B}$ \\
\hline & 100 & $54.6 \mathrm{a}$ & $74.8 \mathrm{~b}$ & $65.1 \mathrm{a}$ & $77.9 \mathrm{~b}$ & $59.8 \mathrm{a}$ & $76.3 \mathrm{~b}$ & $64.7 \mathrm{~B}$ & $71.5 \mathrm{C}$ & $68.1 \mathrm{C}$ \\
\hline & 150 & $76.0 \mathrm{a}$ & $106.3 \mathrm{~b}$ & $87.8 \mathrm{a}$ & $114.7 \mathrm{~b}$ & $81.9 \mathrm{a}$ & $110.5 \mathrm{~b}$ & $91.2 \mathrm{C}$ & 101.3 D & $96.2 \mathrm{D}$ \\
\hline \multirow{4}{*}{3} & 0 & $80.4 \mathrm{a}$ & $128.2 \mathrm{~b}$ & $92.0 \mathrm{a}$ & $128.0 \mathrm{~b}$ & $86.2 \mathrm{a}$ & $128.1 \mathrm{~b}$ & $104.3 \mathrm{~A}$ & $110.0 \mathrm{~A}$ & $107.1 \mathrm{~A}$ \\
\hline & 50 & $76.5 \mathrm{a}$ & $.1 \mathrm{~b}$ & $85.1 \mathrm{a}$ & $115.1 \mathrm{~b}$ & $80.8 \mathrm{a}$ & $113.6 \mathrm{~b}$ & $94.3 \mathrm{~A}$ & $100.1 \mathrm{AB}$ & $97.2 \mathrm{~B}$ \\
\hline & 100 & $107.2 \mathrm{a}$ & $138.4 \mathrm{~b}$ & $80.8 \mathrm{a}$ & $104.9 \mathrm{~b}$ & $94.0 \mathrm{a}$ & $121.6 \mathrm{~b}$ & $122.8 \mathrm{~B}$ & $92.8 \mathrm{~B}$ & $107.8 \mathrm{~A}$ \\
\hline & 15 & $63.7 \mathrm{a}$ & $86.7 \mathrm{~b}$ & $70.6 \mathrm{a}$ & $97.6 \mathrm{~b}$ & $67.2 \mathrm{a}$ & $92.2 \mathrm{~b}$ & $75.2 \mathrm{C}$ & 84.1 BC & $79.7 \mathrm{C}$ \\
\hline \multirow{4}{*}{ Mean } & 0 & $98.9 \mathrm{a}$ & $150.0 \mathrm{~b}$ & $110.4 \mathrm{a}$ & $152.2 \mathrm{~b}$ & $104.7 \mathrm{a}$ & $151.1 \mathrm{~b}$ & $124.5 \mathrm{~A}$ & $131.3 \mathrm{~A}$ & $127.9 \mathrm{~A}$ \\
\hline & 50 & $93.6 \mathrm{a}$ & $133.3 \mathrm{~b}$ & $102.2 \mathrm{a}$ & $133.7 \mathrm{~b}$ & $97.9 \mathrm{a}$ & $133.5 \mathrm{~b}$ & $113.4 \mathrm{AB}$ & $118.0 \mathrm{~B}$ & 115.7 B \\
\hline & 100 & $87.9 \mathrm{a}$ & $115.0 \mathrm{~b}$ & $86.1 \mathrm{a}$ & $107.4 \mathrm{~b}$ & $87.0 \mathrm{a}$ & $111.2 \mathrm{~b}$ & $101.5 \mathrm{~B}$ & $96.7 \mathrm{C}$ & $99.1 \mathrm{C}$ \\
\hline & & $78.0 \mathrm{a}$ & $106.3 \mathrm{~b}$ & $88.7 \mathrm{a}$ & $114.3 \mathrm{~b}$ & $82.4 \mathrm{a}$ & $110.3 \mathrm{~b}$ & $92.2 \mathrm{BC}$ & $100.5 \mathrm{C}$ & $96.3 \mathrm{C}$ \\
\hline 1 & \multirow{3}{*}{ Mean } & $105.6 \mathrm{a}$ & $146.9 \mathrm{~b}$ & $114.1 \mathrm{a}$ & $145.9 \mathrm{~b}$ & $109.8 \mathrm{a}$ & $146.4 \mathrm{~b}$ & $126.2 \mathrm{~A}$ & $130.0 \mathrm{~A}$ & $128.1 \mathrm{~A}$ \\
\hline 2 & & & $115.2 \mathrm{~b}$ & $92.8 \mathrm{a}$ & $123.4 \mathrm{~b}$ & $87.0 \mathrm{a}$ & $119.3 \mathrm{~b}$ & $98.2 \mathrm{~B}$ & $108.1 \mathrm{~B}$ & $103.2 \mathrm{~B}$ \\
\hline 3 & & $81.9 \mathrm{a}$ & $116.3 \mathrm{~b}$ & $82.1 \mathrm{a}$ & $111.4 \mathrm{~b}$ & $82.0 \mathrm{a}$ & $113.9 \mathrm{~b}$ & $99.2 \mathrm{~B}$ & $96.8 \mathrm{C}$ & $98.0 \mathrm{~B}$ \\
\hline & Mean & $89,6 \mathrm{a}$ & $126.1 \mathrm{~b}$ & $96.3 \mathrm{a}$ & $126.9 \mathrm{~b}$ & $92.9 \mathrm{a}$ & $126.5 \mathrm{~b}$ & $107.9 \mathrm{a}$ & $111.6 \mathrm{a}$ & 109.8 \\
\hline
\end{tabular}

Different small letters within the same line indicate significant differences. Values in columns for individual factors indicated with different, capital letters differ significantly

Regardless of the remaining factors, increasing nitrogen rates significantly reduced sugar content in the biomass of the test plants, which has been confirmed by results reported by other authors (Godlewska and Ciepiela, 2018). The highest decline in the Lolium perenne content of monosaccharides (by $32.8 \%$, on average) compared with the biomass harvested in the unit without nitrogen fertiliser was recorded at the rate of $150 \mathrm{~kg} \mathrm{~N} \mathrm{ha}{ }^{-1}$.

Analysis of results obtained for successive cuts revealed that grasses harvested with the second and third cut contained significantly less monosaccharides compared with their contents determined in the first cut plants (average across years). It can be explained by the fact that plant respiration and utilisation of these compounds is higher at high temperatures.

Non-structural and structural carbohydrate contents as well as lignin content are species-related and exerts a substantial effect on the quality of produced feed. Both an excess and shortage of these compounds in feed fed to ruminants is not recommended. Kelpak application had the same extent of a positive effect on cellulose, hemicellulose and lignin contents in both Lolium perenne cultivars (Tables 3, 4 and 5), the contents being by, respectively, 6.64, 8.38 and $8.68 \%$ lower in the biomass of these grasses. The differences were statistically significant. The obtained results disagree with research by Kocira et al. (2018b) who applied a natural sea alga extract-based biostimulant and obtained a significant increase in the soybean seed content of lignin. The aforementioned discrepancy may result from the fact that the effect of natural biostimulants heavily depends on their concentration, application method and, first and foremost, crop plant species and cultivar (Sultana et al., 2005). 
Table 3. Content of cellulose in Lolium perenne ( $\mathrm{g}^{\mathrm{kg}} \mathrm{g}^{-1} \mathrm{~s} . \mathrm{m}$.) depending on biostimulator, nitrogen dose and cut (mean from years 2010-2012)

\begin{tabular}{|c|c|c|c|c|c|c|c|c|c|c|}
\hline \multirow{4}{*}{ Cut } & \multirow{4}{*}{$\begin{array}{c}\text { N dose } \\
\left(\mathrm{kg} \mathrm{N} \mathrm{ha}^{-1}\right)\end{array}$} & \multicolumn{4}{|c|}{ Cultivar } & \multirow{2}{*}{\multicolumn{2}{|c|}{ Mean }} & \multicolumn{2}{|c|}{ Cultivar } & \multirow{4}{*}{ Mean } \\
\hline & & & nent & & & & & Diament & Gagat & \\
\hline & & \multicolumn{4}{|c|}{ Dose of Klepak $\left(\mathrm{dm}^{3} \cdot \mathrm{ha}^{-1}\right)$} & \multicolumn{2}{|c|}{ Dose of Klepak } & \multirow{2}{*}{\multicolumn{2}{|c|}{ Mean }} & \\
\hline & & $\mathbf{0}$ & 2 & 0 & 2 & $\mathbf{0}$ & 2 & & & \\
\hline \multirow{4}{*}{1} & 0 & $287 \mathrm{a}$ & $270 \mathrm{~b}$ & 299 a & $278 \mathrm{~b}$ & $293 \mathrm{a}$ & $274 \mathrm{~b}$ & $279 \mathrm{~A}$ & $289 \mathrm{~A}$ & $284 \mathrm{~A}$ \\
\hline & 50 & $282 \mathrm{a}$ & $265 \mathrm{~b}$ & $289 \mathrm{a}$ & $274 \mathrm{~b}$ & $285 \mathrm{a}$ & $269 \mathrm{~b}$ & $273 \mathrm{AB}$ & $281 \mathrm{AB}$ & $277 \mathrm{AB}$ \\
\hline & 100 & $275 \mathrm{a}$ & $260 \mathrm{~b}$ & $284 \mathrm{a}$ & $266 \mathrm{~b}$ & $279 \mathrm{a}$ & $263 \mathrm{~b}$ & $267 \mathrm{AB}$ & $275 \mathrm{~B}$ & $271 \mathrm{~B}$ \\
\hline & 150 & $268 \mathrm{a}$ & $251 \mathrm{~b}$ & $275 \mathrm{a}$ & $254 \mathrm{~b}$ & $272 \mathrm{a}$ & $252 \mathrm{~b}$ & $260 \mathrm{~B}$ & $265 \mathrm{BC}$ & $262 \mathrm{~B}$ \\
\hline \multirow{4}{*}{2} & 0 & $275 \mathrm{a}$ & $259 \mathrm{~b}$ & $290 \mathrm{a}$ & $267 \mathrm{~b}$ & $283 \mathrm{a}$ & $263 \mathrm{~b}$ & $267 \mathrm{~A}$ & $279 \mathrm{~A}$ & $273 \mathrm{~A}$ \\
\hline & 50 & $271 \mathrm{a}$ & $254 \mathrm{~b}$ & $279 \mathrm{a}$ & $265 \mathrm{a}$ & $275 \mathrm{a}$ & $259 \mathrm{~b}$ & $262 \mathrm{AB}$ & $272 \mathrm{AB}$ & $267 \mathrm{AB}$ \\
\hline & 100 & $265 \mathrm{a}$ & $250 \mathrm{~b}$ & $272 \mathrm{a}$ & $255 \mathrm{~b}$ & $268 \mathrm{a}$ & $253 \mathrm{~b}$ & $257 \mathrm{AB}$ & 264 B & $260 \mathrm{~B}$ \\
\hline & 150 & $259 \mathrm{a}$ & $243 \mathrm{~b}$ & $265 \mathrm{a}$ & $241 \mathrm{~b}$ & $262 \mathrm{a}$ & $242 \mathrm{~b}$ & $251 \mathrm{~B}$ & $253 \mathrm{C}$ & $252 \mathrm{BC}$ \\
\hline \multirow{4}{*}{3} & 0 & $272 \mathrm{a}$ & $255 \mathrm{~b}$ & $278 \mathrm{a}$ & $261 \mathrm{~b}$ & $275 \mathrm{a}$ & $258 \mathrm{~b}$ & $263 \mathrm{~A}$ & $270 \mathrm{~A}$ & $266 \mathrm{~A}$ \\
\hline & 50 & $265 \mathrm{a}$ & $249 \mathrm{~b}$ & $272 \mathrm{a}$ & $255 \mathrm{~b}$ & $268 \mathrm{a}$ & $252 \mathrm{~b}$ & $257 \mathrm{AB}$ & $264 \mathrm{AB}$ & $260 \mathrm{AB}$ \\
\hline & 100 & $254 \mathrm{a}$ & $241 \mathrm{a}$ & $264 \mathrm{a}$ & $248 \mathrm{~b}$ & $259 a$ & $245 \mathrm{~b}$ & $247 \mathrm{~B}$ & $256 \mathrm{~B}$ & $252 \mathrm{~B}$ \\
\hline & 150 & $250 \mathrm{a}$ & $235 \mathrm{~b}$ & $258 \mathrm{a}$ & $243 \mathrm{~b}$ & $254 \mathrm{a}$ & $239 \mathrm{~b}$ & $242 \mathrm{BC}$ & $250 \mathrm{BC}$ & 246BC \\
\hline \multirow{4}{*}{ Mean } & 0 & $278 \mathrm{a}$ & $261 \mathrm{~b}$ & $289 \mathrm{a}$ & $269 \mathrm{~b}$ & $284 \mathrm{a}$ & $265 \mathrm{~b}$ & $270 \mathrm{~A}$ & $279 \mathrm{~A}$ & $274 \mathrm{~A}$ \\
\hline & 50 & $272 a$ & $256 \mathrm{~b}$ & $280 a$ & $265 \mathrm{~b}$ & $276 \mathrm{a}$ & $260 \mathrm{~b}$ & $264 \mathrm{AB}$ & $272 \mathrm{AB}$ & $268 \mathrm{~B}$ \\
\hline & 100 & $264 \mathrm{a}$ & $250 \mathrm{~b}$ & $273 \mathrm{a}$ & $257 \mathrm{~b}$ & $269 \mathrm{a}$ & $253 \mathrm{~b}$ & $257 \mathrm{~B}$ & 265 B & $261 \mathrm{C}$ \\
\hline & & $259 \mathrm{a}$ & $243 \mathrm{~b}$ & $266 \mathrm{a}$ & $246 \mathrm{~b}$ & $263 \mathrm{a}$ & $244 \mathrm{~b}$ & $251 \mathrm{BC}$ & $256 \mathrm{C}$ & $253 \mathrm{D}$ \\
\hline 1 & \multirow{3}{*}{ Mean } & $278 \mathrm{a}$ & $261 \mathrm{~b}$ & $287 \mathrm{a}$ & $268 \mathrm{~b}$ & $282 \mathrm{a}$ & $265 \mathrm{~b}$ & $270 \mathrm{~A}$ & 277 A & $274 \mathrm{~A}$ \\
\hline 2 & & $268 \mathrm{a}$ & $251 \mathrm{~b}$ & $276 \mathrm{a}$ & $257 \mathrm{~b}$ & $272 \mathrm{a}$ & $254 \mathrm{~b}$ & $259 \mathrm{~B}$ & $267 \mathrm{~B}$ & $263 \mathrm{~B}$ \\
\hline 3 & & $260 a$ & $245 \mathrm{~b}$ & $268 \mathrm{a}$ & $252 \mathrm{~b}$ & $264 \mathrm{a}$ & $248 \mathrm{~b}$ & $252 \mathrm{~B}$ & $260 \mathrm{~B}$ & $256 \mathrm{C}$ \\
\hline \multicolumn{2}{|r|}{ Mean } & $268 \mathrm{a}$ & $253 \mathrm{~b}$ & $277 \mathrm{a}$ & $259 \mathrm{~b}$ & $273 \mathrm{a}$ & $256 \mathrm{~b}$ & $260 \mathrm{a}$ & $268 \mathrm{a}$ & 264 \\
\hline
\end{tabular}

Different small letters within the same line indicate significant differences. Values in columns for individual factors indicated with different, capital letters differ significantly

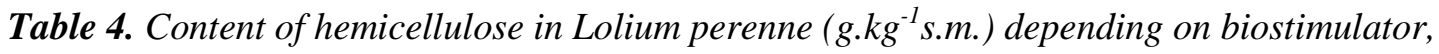
nitrogen dose and cut (mean from years 2010-2012)

\begin{tabular}{|c|c|c|c|c|c|c|c|c|c|c|}
\hline \multirow{4}{*}{ Cut } & \multirow{4}{*}{$\begin{array}{c}\mathbf{N} \text { dose } \\
\left(\mathbf{k g ~ N} \mathbf{N h a}^{-1}\right)\end{array}$} & \multicolumn{4}{|c|}{ Cultivar } & \multirow{2}{*}{\multicolumn{2}{|c|}{ Mean }} & \multicolumn{2}{|c|}{ Cultivar } & \multirow{4}{*}{ Mean } \\
\hline & & Dia & & & & & & Diament & Gagat & \\
\hline & & \multicolumn{4}{|c|}{ Dose of Klepak $\left(\mathrm{dm}^{3} \bullet \mathrm{ha}^{-1}\right)$} & Dose o & Klepak & \multirow{2}{*}{\multicolumn{2}{|c|}{ Mean }} & \\
\hline & & $\mathbf{0}$ & 2 & $\mathbf{0}$ & 2 & $\mathbf{0}$ & 2 & & & \\
\hline \multirow{4}{*}{1} & 0 & $185 \mathrm{a}$ & $167 \mathrm{~b}$ & $189 \mathrm{a}$ & $177 \mathrm{~b}$ & $187 \mathrm{a}$ & $172 \mathrm{~b}$ & $176 \mathrm{~A}$ & $183 \mathrm{~A}$ & $180 \mathrm{~A}$ \\
\hline & 50 & $175 \mathrm{a}$ & $162 \mathrm{~b}$ & $184 \mathrm{a}$ & $173 b$ & $179 \mathrm{a}$ & $167 \mathrm{~b}$ & $168 \mathrm{~A}$ & $178 \mathrm{~A}$ & $173 \mathrm{~A}$ \\
\hline & 100 & $161 \mathrm{a}$ & $148 \mathrm{~b}$ & $172 \mathrm{a}$ & $155 \mathrm{~b}$ & $166 \mathrm{a}$ & $152 b$ & $155 \mathrm{~B}$ & $164 \mathrm{~B}$ & $159 \mathrm{~B}$ \\
\hline & 150 & $146 \mathrm{a}$ & $130 \mathrm{~b}$ & $147 \mathrm{a}$ & $124 \mathrm{~b}$ & $147 \mathrm{a}$ & $127 \mathrm{~b}$ & $138 \mathrm{C}$ & $135 \mathrm{C}$ & $137 \mathrm{C}$ \\
\hline \multirow{4}{*}{2} & 0 & $195 \mathrm{a}$ & $179 \mathrm{~b}$ & $200 \mathrm{a}$ & $185 \mathrm{~b}$ & $198 \mathrm{a}$ & $182 \mathrm{~b}$ & $187 \mathrm{~A}$ & $192 \mathrm{~A}$ & $190 \mathrm{~A}$ \\
\hline & 50 & $186 \mathrm{a}$ & $175 \mathrm{~b}$ & $193 \mathrm{a}$ & $181 \mathrm{~b}$ & $190 \mathrm{a}$ & $178 \mathrm{~b}$ & $180 \mathrm{AB}$ & $187 \mathrm{~A}$ & $184 \mathrm{AB}$ \\
\hline & 100 & $181 \mathrm{a}$ & $170 \mathrm{~b}$ & $188 \mathrm{a}$ & $179 \mathrm{a}$ & $185 \mathrm{a}$ & $175 \mathrm{~b}$ & $175 \mathrm{~B}$ & $184 \mathrm{AB}$ & $180 \mathrm{~B}$ \\
\hline & 150 & $175 \mathrm{a}$ & $166 \mathrm{a}$ & $186 \mathrm{a}$ & $163 \mathrm{~b}$ & $181 \mathrm{a}$ & $165 \mathrm{~b}$ & $171 \mathrm{~B}$ & $175 \mathrm{~B}$ & $173 \mathrm{BC}$ \\
\hline \multirow{4}{*}{3} & 0 & $191 \mathrm{a}$ & $176 \mathrm{~b}$ & $188 \mathrm{a}$ & $177 \mathrm{~b}$ & $190 \mathrm{a}$ & $177 \mathrm{~b}$ & $184 \mathrm{~A}$ & $183 \mathrm{~A}$ & $183 \mathrm{~A}$ \\
\hline & 50 & $180 \mathrm{a}$ & $170 \mathrm{a}$ & $187 \mathrm{a}$ & $175 \mathrm{~b}$ & $184 \mathrm{a}$ & $173 \mathrm{~b}$ & $175 \mathrm{AB}$ & $181 \mathrm{AB}$ & $178 \mathrm{~A}$ \\
\hline & 100 & $173 \mathrm{a}$ & $160 \mathrm{~b}$ & $177 \mathrm{a}$ & $166 \mathrm{~b}$ & $175 \mathrm{a}$ & $163 \mathrm{~b}$ & $166 \mathrm{~B}$ & $172 \mathrm{~B}$ & $169 \mathrm{~B}$ \\
\hline & 150 & $163 \mathrm{a}$ & $136 \mathrm{~b}$ & $168 \mathrm{a}$ & $152 \mathrm{~b}$ & $165 \mathrm{a}$ & $144 \mathrm{~b}$ & $150 \mathrm{C}$ & $160 \mathrm{C}$ & $155 \mathrm{C}$ \\
\hline \multirow{4}{*}{ Mean } & 0 & $190 \mathrm{a}$ & $174 \mathrm{~b}$ & $192 \mathrm{a}$ & $180 \mathrm{~b}$ & $192 \mathrm{a}$ & $177 \mathrm{~b}$ & $184 \mathrm{~A}$ & $186 \mathrm{~A}$ & $184 \mathrm{~A}$ \\
\hline & 50 & $180 \mathrm{a}$ & $169 \mathrm{~b}$ & $188 \mathrm{a}$ & $176 \mathrm{~b}$ & $185 \mathrm{a}$ & $173 \mathrm{~b}$ & $175 \mathrm{~B}$ & $182 \mathrm{~A}$ & $179 \mathrm{~A}$ \\
\hline & 100 & $172 \mathrm{a}$ & $159 \mathrm{~b}$ & $179 \mathrm{a}$ & $167 \mathrm{~b}$ & $176 \mathrm{a}$ & $163 \mathrm{~b}$ & $166 \mathrm{C}$ & $173 \mathrm{~B}$ & $169 \mathrm{~B}$ \\
\hline & 150 & $161 \mathrm{a}$ & $144 \mathrm{~b}$ & $167 \mathrm{a}$ & $146 \mathrm{~b}$ & $165 \mathrm{a}$ & $144 \mathrm{~b}$ & $153 \mathrm{D}$ & $157 \mathrm{C}$ & $155 \mathrm{C}$ \\
\hline 1 & \multirow{3}{*}{ Mean } & $167 \mathrm{a}$ & $152 \mathrm{~b}$ & $173 \mathrm{a}$ & $157 \mathrm{~b}$ & $170 \mathrm{a}$ & $155 \mathrm{~b}$ & $159 \mathrm{~A}$ & $165 \mathrm{~A}$ & $162 \mathrm{~A}$ \\
\hline 2 & & $185 \mathrm{a}$ & $173 a$ & $192 \mathrm{a}$ & $177 \mathrm{~b}$ & $188 \mathrm{a}$ & $175 \mathrm{~b}$ & $179 \mathrm{~B}$ & $184 \mathrm{~B}$ & $181 \mathrm{~B}$ \\
\hline 3 & & $177 \mathrm{a}$ & $161 \mathrm{~b}$ & $180 \mathrm{a}$ & $168 \mathrm{a}$ & $178 \mathrm{a}$ & $164 \mathrm{~b}$ & $169 \mathrm{AB}$ & $174 \mathrm{AB}$ & $171 \mathrm{C}$ \\
\hline & Mean & $176 \mathrm{a}$ & $162 \mathrm{~b}$ & $182 \mathrm{a}$ & $167 \mathrm{~b}$ & $179 \mathrm{a}$ & $164 \mathrm{~b}$ & $169 \mathrm{a}$ & $174 \mathrm{a}$ & 172 \\
\hline
\end{tabular}

Different small letters within the same line indicate significant differences. Values in columns for individual factors indicated with different, capital letters differ significantly 
Table 5. Content of lignin in Lolium perenne ( $\mathrm{kg}^{-1}$ s.m. $)$ depending on biostimulator, nitrogen dose and cut (mean from years 2010-2012)

\begin{tabular}{|c|c|c|c|c|c|c|c|c|c|c|}
\hline \multirow{4}{*}{ Cut } & \multirow{4}{*}{$\begin{array}{c}\text { N dose } \\
\left(k^{\prime} \text { N.ha }^{-1}\right)\end{array}$} & \multicolumn{4}{|c|}{ Cultivar } & \multirow{2}{*}{\multicolumn{2}{|c|}{ Mean }} & \multicolumn{2}{|c|}{ Cultivar } & \multirow{4}{*}{ Mean } \\
\hline & & \multicolumn{2}{|l|}{ Diament } & \multicolumn{2}{|c|}{ Gagat } & & & \multirow{3}{*}{\multicolumn{2}{|c|}{\begin{tabular}{r|} 
Diament \\
Mea
\end{tabular}}} & \\
\hline & & \multicolumn{4}{|c|}{$\begin{array}{l}\text { Dose of Klepak } \\
\left(\mathrm{dm}^{3} \cdot \mathrm{ha}^{-1}\right)\end{array}$} & \multicolumn{2}{|c|}{$\begin{array}{c}\text { Dose of Klepak } \\
\left(\mathbf{d m}^{3} \cdot \mathbf{h a}^{-1}\right)\end{array}$} & & & \\
\hline & & $\mathbf{0}$ & 2 & 0 & 2 & 0 & 2 & & & \\
\hline \multirow{4}{*}{1} & 0 & $40.1 \mathrm{a}$ & $37.0 \mathrm{~b}$ & $41.2 \mathrm{a}$ & $37.7 \mathrm{~b}$ & $40.6 \mathrm{a}$ & $37.4 \mathrm{~b}$ & $38.5 \mathrm{~A}$ & $39.5 \mathrm{~A}$ & $39.0 \mathrm{~A}$ \\
\hline & 50 & $38.2 \mathrm{a}$ & $36.1 \mathrm{a}$ & $40.0 \mathrm{a}$ & $36.7 \mathrm{~b}$ & $39.1 \mathrm{a}$ & $36.4 \mathrm{~b}$ & $37.1 \mathrm{AB}$ & $38.4 \mathrm{~A}$ & $37.8 \mathrm{AB}$ \\
\hline & 100 & $37.7 \mathrm{a}$ & $34.8 \mathrm{~b}$ & $38.6 \mathrm{a}$ & $35.8 \mathrm{~b}$ & $38.2 \mathrm{a}$ & $35.3 \mathrm{~b}$ & $36.3 \mathrm{AB}$ & $37.2 \mathrm{AB}$ & $36.8 \mathrm{AB}$ \\
\hline & 150 & $36.7 \mathrm{a}$ & $34.1 \mathrm{~b}$ & $37.7 \mathrm{a}$ & $34.2 \mathrm{~b}$ & $37.2 \mathrm{a}$ & $34.1 \mathrm{~b}$ & $35.4 \mathrm{~B}$ & $36.0 \mathrm{~B}$ & $35.7 \mathrm{~B}$ \\
\hline \multirow{4}{*}{2} & 0 & $41.7 \mathrm{a}$ & $38.4 \mathrm{~b}$ & $44.7 \mathrm{a}$ & $40.0 \mathrm{~b}$ & $43.2 \mathrm{a}$ & $39.2 \mathrm{~b}$ & $40.0 \mathrm{~A}$ & $42.4 \mathrm{~A}$ & $41.2 \mathrm{~A}$ \\
\hline & 50 & $40.1 \mathrm{a}$ & $38.2 \mathrm{a}$ & $42.0 \mathrm{a}$ & $38.4 \mathrm{~b}$ & $41.0 \mathrm{a}$ & $38.3 \mathrm{~b}$ & $39.1 \mathrm{AB}$ & $40.2 \mathrm{AB}$ & $39.7 \mathrm{AB}$ \\
\hline & 100 & $39.2 \mathrm{a}$ & $36.5 \mathrm{~b}$ & $39.8 \mathrm{a}$ & $36.7 \mathrm{~b}$ & $39.5 \mathrm{a}$ & $36.6 \mathrm{~b}$ & $37.8 \mathrm{AB}$ & $38.3 \mathrm{~B}$ & $38.0 \mathrm{~B}$ \\
\hline & 150 & $38.7 \mathrm{a}$ & $35.2 \mathrm{~b}$ & $39.0 \mathrm{a}$ & $35.5 \mathrm{~b}$ & $38.9 \mathrm{a}$ & $35.4 \mathrm{~b}$ & $37.0 \mathrm{~B}$ & $37.3 \mathrm{BC}$ & $37.1 \mathrm{BC}$ \\
\hline \multirow{4}{*}{3} & 0 & $38.4 \mathrm{a}$ & $35.3 \mathrm{~b}$ & $39.8 \mathrm{a}$ & $36.3 \mathrm{~b}$ & $39.1 \mathrm{a}$ & $35.8 \mathrm{~b}$ & $36.8 \mathrm{~A}$ & $38.0 \mathrm{~A}$ & $37.4 \mathrm{~A}$ \\
\hline & 50 & $36.4 \mathrm{a}$ & $34.5 \mathrm{~b}$ & $38.2 \mathrm{a}$ & $34.5 \mathrm{~b}$ & $37.3 \mathrm{a}$ & $34.5 \mathrm{~b}$ & $35.4 \mathrm{AB}$ & $36.4 \mathrm{AB}$ & $35.9 \mathrm{AB}$ \\
\hline & 100 & $35.5 \mathrm{a}$ & $33.3 \mathrm{a}$ & $36.1 \mathrm{a}$ & $33.8 \mathrm{a}$ & $35.8 \mathrm{a}$ & $33.5 \mathrm{a}$ & $34.4 \mathrm{~B}$ & $35.0 \mathrm{~B}$ & 34.7 B \\
\hline & 150 & $35.0 \mathrm{a}$ & $31.7 \mathrm{~b}$ & $36.0 \mathrm{a}$ & $32.5 \mathrm{~b}$ & $35.5 \mathrm{a}$ & $32.1 \mathrm{~b}$ & $33.4 \mathrm{~B}$ & $34.2 \mathrm{~B}$ & $33.8 \mathrm{~B}$ \\
\hline \multirow{4}{*}{ Mean } & 0 & $40.1 \mathrm{a}$ & $36.9 \mathrm{~b}$ & $41.9 \mathrm{a}$ & $38.0 \mathrm{~b}$ & $41.0 \mathrm{a}$ & $37.4 \mathrm{~b}$ & $38.5 \mathrm{~A}$ & $40.0 \mathrm{~A}$ & $39.2 \mathrm{~A}$ \\
\hline & 50 & $38.2 \mathrm{a}$ & $36.2 \mathrm{a}$ & $40.1 \mathrm{a}$ & $36.5 \mathrm{~b}$ & $39.1 \mathrm{a}$ & $36.4 \mathrm{~b}$ & $37.2 \mathrm{AB}$ & $38.3 \mathrm{AB}$ & $37.8 \mathrm{AB}$ \\
\hline & 100 & $37.5 \mathrm{a}$ & $34.8 \mathrm{~b}$ & $38.2 \mathrm{a}$ & $35.5 \mathrm{~b}$ & $37.8 \mathrm{a}$ & $35.2 \mathrm{~b}$ & $36.2 \mathrm{~B}$ & 36.8 B & $36.5 \mathrm{~B}$ \\
\hline & 150 & $36.8 \mathrm{a}$ & $33.7 \mathrm{~b}$ & $37.6 \mathrm{a}$ & $34.1 \mathrm{~b}$ & $37.2 \mathrm{a}$ & $33.9 \mathrm{~b}$ & $35.2 \mathrm{~B}$ & $35.8 \mathrm{BC}$ & $35.5 \mathrm{BC}$ \\
\hline 1 & \multirow{3}{*}{ Mean } & $38.2 \mathrm{a}$ & $35.5 \mathrm{~b}$ & $39.4 \mathrm{a}$ & $36.1 \mathrm{~b}$ & $38.8 \mathrm{a}$ & $35.8 \mathrm{~b}$ & $36.8 \mathrm{~A}$ & $37.8 \mathrm{~A}$ & $37.3 \mathrm{~A}$ \\
\hline 2 & & $39.9 \mathrm{a}$ & $37.0 \mathrm{~b}$ & $41.4 \mathrm{a}$ & $37.7 \mathrm{~b}$ & $40.7 \mathrm{a}$ & $37.4 \mathrm{~b}$ & $38.5 \mathrm{AB}$ & $39.5 \mathrm{~A}$ & $39.0 \mathrm{~A}$ \\
\hline 3 & & $36.3 \mathrm{a}$ & $33.7 \mathrm{~b}$ & $37.5 \mathrm{a}$ & $34.3 \mathrm{~b}$ & $36.9 \mathrm{a}$ & $34.0 \mathrm{~b}$ & $35.0 \mathrm{AC}$ & $35.9 \mathrm{~B}$ & $35.4 \mathrm{~B}$ \\
\hline & Iean & $38.1 \mathrm{a}$ & $35.4 \mathrm{~b}$ & $39.4 \mathrm{a}$ & $36.0 \mathrm{~b}$ & $38.8 \mathrm{a}$ & $35.7 \mathrm{~b}$ & $36.8 \mathrm{a}$ & $37.7 \mathrm{a}$ & 37.2 \\
\hline
\end{tabular}

Different small letters within the same line indicate significant differences. Values in columns for individual factors indicated with different, capital letters differ significantly

An interaction of sea alga extract and nitrogen fertilisation was consistently in the direction of reducing the concentration of structural carbohydrates and lignin in both Lolium perenne cultivars treated with the biostimulant at each nitrogen fertiliser rate. The strongest interaction was recorded in the plots fertilised with $150 \mathrm{~kg} \mathrm{~N} \mathrm{ha}^{-1}$ where Kelpak application contributed to a decline in the Lolium perenne content of cellulose, hemicellulose and lignin (respectively, by 7.79, 14.6 and 9.74\%). Unfortunately, it is impossible to refer these results to other reports due to lack of literature on this issue.

Regardless of the remaining factors, nitrogen fertilisation contributed to a decline in the Lolium perenne content of the discussed compounds. Cellulose and hemicellulose contents in grass biomass declined as nitrogen rates increased, the differences being statistically significant. Similar tendencies were observed for lignin content but significant differences were found only between lignin content in plants harvested from plots without nitrogen fertiliser, and plants fertilised with the nitrogen rate of $150 \mathrm{~kg}^{-1} \mathrm{ha}^{-1}$. According to Szkutnik et al. (2012) as well as Ciepiela et al. (2016), high nitrogen rates contribute to a decline in the grass content of raw fibre, which is consistent with the study results reported here.

The concentration of cellulose and hemicellulose in grasses depended significantly on cuts, and differences in the contents of these compounds in the test grass biomass harvested with individual cuts were significant.

Lolium perenne cv. Diament contained less structural carbohydrates and lignin than cv. Gagat but the differences were statistically insignificant. 
Non-structural carbohydrate content in both Lolium perenne cultivars as affected by the biostimulant, nitrogen fertilisation and cuts is shown in Table 6. Statistical analysis of the study results demonstrated a significant influence of all the experimental factors on non-structural carbohydrate content in the test plant material. The results indicate that there was a uniform positive effect of the biostimulant on the Lolium perenne content of the discussed compounds. Grasses treated with Kelpak contained by $9.79 \%$ more non-structural carbohydrates compared with their concentration in the biomass of plants which had not been treated with the biostimulant. As far as an interaction between Ecklonia maxima extract and nitrogen rates is concerned, there was found a significantly higher non-structural carbohydrate content in both grass species treated with the biostimulant and at each nitrogen rate. The lowest increase in the concentration of the compounds following Kelpak application, amounting to $8.15 \%$, was found in the biomass of plants fertilised with the rate of $50 \mathrm{~kg} \mathrm{~N} \mathrm{ha}^{-1}$, it being the highest $(11.9 \%)$ in the biomass of plants treated with the biostimulant and fertilised with nitrogen at the rate of $150 \mathrm{~kg} \cdot \mathrm{ha}^{1}$.

Table 6. Content of non-structural carbohydrates in Lolium perenne (g. $\mathrm{kg}^{-1}$ s.m.) depending on biostimulator, nitrogen dose and cut (mean from years 2010-2012)

\begin{tabular}{|c|c|c|c|c|c|c|c|c|c|c|}
\hline \multirow{4}{*}{ Cut } & \multirow{4}{*}{$\begin{array}{c}\text { N dose } \\
\left(\mathrm{kg} \mathrm{N} \cdot h \mathrm{a}^{-1}\right)\end{array}$} & \multicolumn{4}{|c|}{ Cultivar } & \multirow{2}{*}{\multicolumn{2}{|c|}{ Mean }} & \multicolumn{2}{|c|}{ Cultivar } & \multirow{4}{*}{ Mean } \\
\hline & & \multicolumn{2}{|c|}{ Diament } & \multicolumn{2}{|c|}{ Gagat } & & & Diament & Gagat & \\
\hline & & \multicolumn{4}{|c|}{$\begin{array}{c}\text { Dose of Klepak } \\
\left(\mathbf{d m}^{3} \cdot \mathbf{h a}^{-1}\right)\end{array}$} & \multicolumn{2}{|c|}{$\begin{array}{c}\text { Dose of Klepak } \\
\left(\mathbf{d m}^{3} \cdot \mathrm{ha}^{-1}\right)\end{array}$} & \multirow{2}{*}{\multicolumn{2}{|c|}{ Mean }} & \\
\hline & & $\mathbf{0}$ & 2 & $\mathbf{0}$ & 2 & 0 & 2 & & & \\
\hline \multirow{4}{*}{1} & 0 & $278 \mathrm{a}$ & $303 \mathrm{~b}$ & $262 \mathrm{a}$ & $287 \mathrm{~b}$ & $270 \mathrm{a}$ & $295 \mathrm{~b}$ & $291 \mathrm{~A}$ & $274 \mathrm{~A}$ & $283 \mathrm{~A}$ \\
\hline & 50 & $274 a$ & $296 \mathrm{~b}$ & $253 a$ & $275 \mathrm{~b}$ & $263 a$ & $286 \mathrm{~b}$ & $285 \mathrm{~A}$ & $264 \mathrm{AB}$ & $274 \mathrm{AB}$ \\
\hline & 100 & $271 \mathrm{a}$ & $289 a$ & $245 \mathrm{a}$ & $269 \mathrm{~b}$ & $258 \mathrm{a}$ & $279 \mathrm{~b}$ & $280 \mathrm{~A}$ & $257 \mathrm{~B}$ & $269 \mathrm{~B}$ \\
\hline & 150 & $257 \mathrm{a}$ & $281 \mathrm{~b}$ & $241 \mathrm{a}$ & $280 \mathrm{~b}$ & $249 \mathrm{a}$ & $280 \mathrm{~b}$ & $269 \mathrm{~B}$ & $260 \mathrm{~B}$ & $265 \mathrm{~B}$ \\
\hline \multirow{4}{*}{2} & 0 & $240 \mathrm{a}$ & $257 \mathrm{a}$ & $227 \mathrm{a}$ & $240 \mathrm{a}$ & $233 \mathrm{a}$ & $248 \mathrm{a}$ & $248 \mathrm{~A}$ & $233 \mathrm{~A}$ & $241 \mathrm{~A}$ \\
\hline & 50 & $221 \mathrm{a}$ & $241 \mathrm{~b}$ & $205 \mathrm{a}$ & $223 \mathrm{a}$ & $213 \mathrm{a}$ & $232 \mathrm{a}$ & $231 \mathrm{~B}$ & $214 \mathrm{~B}$ & $222 \mathrm{~B}$ \\
\hline & 100 & $215 \mathrm{a}$ & $232 a$ & $197 \mathrm{a}$ & $217 \mathrm{~b}$ & $206 \mathrm{a}$ & $225 \mathrm{a}$ & $224 \mathrm{~B}$ & $207 \mathrm{BC}$ & $215 \mathrm{~B}$ \\
\hline & 150 & $191 \mathrm{a}$ & $222 \mathrm{~b}$ & $186 \mathrm{a}$ & $204 \mathrm{a}$ & $189 \mathrm{a}$ & $213 \mathrm{~b}$ & $207 \mathrm{C}$ & $195 \mathrm{C}$ & $201 \mathrm{C}$ \\
\hline \multirow{4}{*}{3} & 0 & $268 \mathrm{a}$ & $291 \mathrm{~b}$ & $237 \mathrm{a}$ & $275 b$ & $253 \mathrm{a}$ & $283 \mathrm{~b}$ & $280 \mathrm{~A}$ & $256 \mathrm{~A}$ & $268 \mathrm{~A}$ \\
\hline & 50 & $244 \mathrm{a}$ & $261 \mathrm{a}$ & $229 a$ & $255 \mathrm{~b}$ & $237 \mathrm{a}$ & $258 \mathrm{~b}$ & $253 \mathrm{~B}$ & 242 B & 247 B \\
\hline & 100 & $245 \mathrm{a}$ & $261 \mathrm{a}$ & $221 \mathrm{a}$ & $243 \mathrm{~b}$ & $233 \mathrm{a}$ & $252 \mathrm{a}$ & $253 \mathrm{~B}$ & $232 \mathrm{BC}$ & $242 B C$ \\
\hline & 150 & $222 \mathrm{a}$ & $243 b$ & $218 \mathrm{a}$ & $240 \mathrm{~b}$ & $220 \mathrm{a}$ & $241 \mathrm{~b}$ & $232 \mathrm{C}$ & $229 \mathrm{C}$ & $231 \mathrm{C}$ \\
\hline \multirow{4}{*}{ Mean } & 0 & $262 \mathrm{a}$ & $284 \mathrm{~b}$ & $242 a$ & $267 \mathrm{~b}$ & $252 \mathrm{a}$ & $276 \mathrm{~b}$ & $273 \mathrm{~A}$ & $254 \mathrm{~A}$ & $264 \mathrm{~A}$ \\
\hline & 50 & $246 \mathrm{a}$ & $266 \mathrm{~b}$ & $229 a$ & $251 \mathrm{~b}$ & $238 \mathrm{a}$ & $258 \mathrm{~b}$ & $256 \mathrm{~B}$ & $240 \mathrm{~B}$ & $248 \mathrm{~B}$ \\
\hline & 100 & $244 \mathrm{a}$ & $261 \mathrm{a}$ & $221 \mathrm{a}$ & $243 \mathrm{~b}$ & $232 \mathrm{a}$ & $252 \mathrm{~b}$ & $252 \mathrm{~B}$ & $232 \mathrm{~B}$ & $242 B C$ \\
\hline & 150 & $223 \mathrm{a}$ & $249 \mathrm{~b}$ & $215 \mathrm{a}$ & $241 \mathrm{~b}$ & $219 a$ & $245 \mathrm{~b}$ & $236 \mathrm{C}$ & $228 \mathrm{~B}$ & $232 \mathrm{C}$ \\
\hline 1 & \multirow{3}{*}{ Mean } & $270 \mathrm{a}$ & $292 b$ & $250 \mathrm{a}$ & $278 \mathrm{~b}$ & $260 \mathrm{a}$ & $285 \mathrm{~b}$ & $281 \mathrm{~A}$ & $264 \mathrm{~A}$ & $273 \mathrm{~A}$ \\
\hline 2 & & $217 \mathrm{a}$ & $238 \mathrm{~b}$ & $204 \mathrm{a}$ & $221 \mathrm{a}$ & $210 \mathrm{a}$ & $230 \mathrm{a}$ & $227 \mathrm{~B}$ & $212 \mathrm{~B}$ & $220 \mathrm{~B}$ \\
\hline 3 & & $245 \mathrm{a}$ & $264 \mathrm{~b}$ & $226 \mathrm{a}$ & $253 \mathrm{~b}$ & $236 \mathrm{a}$ & $259 \mathrm{~b}$ & $254 \mathrm{C}$ & $240 \mathrm{C}$ & $247 \mathrm{C}$ \\
\hline \multicolumn{2}{|r|}{ Mean } & $244 \mathrm{a}$ & $265 \mathrm{~b}$ & $227 \mathrm{a}$ & $251 \mathrm{~b}$ & $235 \mathrm{a}$ & $258 \mathrm{~b}$ & $254 \mathrm{a}$ & $239 \mathrm{~b}$ & 246 \\
\hline
\end{tabular}

Different small letters within the same line indicate significant differences. Values in columns for individual factors indicated with different, capital letters differ significantly

Regardless of the remaining factors, increasing nitrogen rates reduced the concentration of non-structural carbohydrates in grass biomass, the majority of the differences being statistically significant. The only insignificant differences were recorded between Lolium perenne biomass contents of the discussed compounds determined in plots fertilised with the nitrogen rates of 50 and $100 \mathrm{~kg} \mathrm{ha}^{-1}$.

Seasonal changes in the concentration of non-structural carbohydrates in the biomass of the harvested plants were observed, the differences being statistically significant. The 
lowest amounts of non-structural carbohydrates were determined in plants harvested with the second cut, them being the highest in the first cut, the differences being significant. Similar relationships were reported by Ciepiela and Godlewska (2015) for orchard grass and Braun's festulolium. As far as Lolium perenne cultivars are concerned, cv. Diament were found to contain by $6.28 \%$ more non-structural carbohydrates compared with their content determined in cv. Gagat, the difference being significant.

\section{Conclusions}

The research reported here demonstrated a positive effect of Ecklonia maxima extract on Lolium perenne chemical composition. Its results showed that structural carbohydrate and lignin contents in grass biomass were reduced whereas non-structural carbohydrate content increased by means of an application of sea alga extract, which results in an improved quality of feed produced from these grasses. The biostimulant applied in combination with each nitrogen rate significantly increased the concentration of monosaccharides and non-structural carbohydrates, and reduced the concentration of structural carbohydrates and lignin in Lolium perenne biomass. Increasing nitrogen rates significantly increased cellulose, hemicellulose and lignin contents but they reduced amounts of monosaccharides and non-structural carbohydrates in the test grasses. The concentration of the examined components in the biomass of Lolium perenne plants clearly varied during the growing season. Further studies are needed to develop more comprehensive results and evaluate the impact of different rates of biostimulants.

Acknowledgments. The results of the research carried out under the research theme No. 41/20/B were financed from the science grant granted by the Ministry of Science and Higher Education.

\section{REFERENCES}

[1] Battacharyya, D., Babgohari, M. Z., Rathor, P., Prithiviraj, B. (2015): Seaweed extracts as biostimulants in horticulture. - Scientia Horticulturae 196: 39-48.

doi.:10.1016/j.scienta.2015.09.012.

[2] Caruso, G., De Pascale, S., Cozzolino, E., Giordano, M., El-Nakhel, C., Cuciniello, A., Cenvinzo, V., Colla, G., Rouphael, Y. (2019): Protein hydrolysate or plant extract-based biostimulants enhanced yield and quality performances of greenhouse perennial wall rocket grown in different seasons. - Plants 8: 208. doi.:10.3390/plants8070208.

[3] Ciepiela, G. A., Godlewska, A. (2015): The effect of growth regulator on structural and non-structural carbohydrates and lignin content in selected grass species and cultivars. Journal of Ecological Engineering 16(3): 189-197. doi.:10.12911/22998993/2955.

[4] Ciepiela, G. A., Godlewska, A., Jankowska, J. (2016): The effect of seaweed Ecklonia maxima and mineral nitrogen on fodder grass chemical composition. - Environmental Science and Pollution Research 23: 2301-2307. doi.:10.1007/s11356-015-5417-3.

[5] COBORU (2008): Wyniki porejestrowych doświadczeń odmianowych. - Kostrzewa łąkowa, życica trwała, życica wielokwiatowa, No 59, Slupia Wielka.

[6] Colla, G., Rouphael, Y. (2015): Biostimulants in horticulture. - Scientia Horticulturae 196: $1-2$.

[7] Du Jardin, P. (2015): Plant biostimulants: definition, concept, main categories and regulation. - Scientia Horticulturae 196: 3-14. doi.:10.1016/j.scienta.2015.09.021. 
[8] El-Miniawy, S. M., Ragab, M. E., Youssef, S. M., Metwally, A. A. (2014): Influence of foliar spraying of seaweed extract on growth, yield and quality of strawberry plants. Journal of Applied Science Research 10(2): 88-94.

[9] Godlewska, A., Ciepiela, G. A. (2013): The effect of natural growth regulators obtained from Ecklonia maxima and mineral nitrogen on true protein and simple sugar contents of Dactylis glomerata L. and Festulolium braunii (K. Richt) A. Camus. - Turkish Journal of Field Crops 18(2): 247-253.

[10] Godlewska, A., Ciepiela, G. A. (2016): The effect of growth regulator on dry matter yield and some chemical components in selected grass species and cultivars. - Soil Science and Plant Nutrition 62(3): 297-302. doi.:10.1080/00380768.2016.1185741.

[11] Godlewska, A., Ciepiela, G. A. (2018): Assessment of the effect of various biostimulants on Medicago x varia T. Martyn yielding and content of selected organic components. Applied Ecology and Environmental Research 16(5): 5571-5581. doi.:10.15666/aeer/1605_55715581.

[12] Haider, M. W., Ayyub, C. H. M., Pervez, M. A., Asad, H. U., Manan, A., Raza, S. A., Ashraf, I. (2012): Impact of foliar application of seaweed extract on growth, yield and quality of potato (Solanum tuberosum L.). - Soil and Environment 31(2): 157-162.

[13] Kocira, S., Szparaga, A., Kocira, A., Czerwińska, E., Depo, K., Erlichowska, B., Deszcz, E. (2018a): Effect of applying a biostimulant containing seaweed and amino acids on the content of fiber fractions in three soybean cultivars. - Legume Research 42(3): 341-347. doi.:10.18805/LR-412.

[14] Kocira, S., Szparaga, A., Kocira, A., Czerwińska, E., Wójtowicz, A., BronowickaMielniczuk, U., Koszel, M., Findura, P. (2018b): Modeling Biometric Traits, Yield and Nutritional and Antioxidant Properties of Seeds of Three Soybean Cultivars Through the Application of Biostimulant Containing Seaweed and Amino Acids. - Frontiers and Plant Science 9(388). doi.:10.3389/fpls.2018.00388.

[15] Niewiadomska, A., Sulewska, H., Wolna-Maruwka, A., Ratajczak, K., Waraczewska, Z., Budka, A. (2020): The influence of bio-stimulants and foliar fertilizers on yield, plant features, and the level of soil biochemical activity in white lupine (Lupinus albus L.) cultivation. - Agronomy 10(1): 150.

[16] Pacholczak, A., Szydło, W., Jacygard, E., Federowicz, M. (2012): Effect of auxins and the biostimulator algaminoplant on rhizogenesis in stem cuttings of two dogwood cultivars (Cornus alba 'Aurea' and 'Elegantissima'). - Acta Scientiarum Polonorum -Hortorum 11(2): 93-103.

[17] Papenfus, H. B., Stirk, W. A., Finnie, J. F., Van Staden, J. (2012): Seasonal variation in the polyamines of Ecklonia maxima. - Botanica Marina 55: 539-546. doi.:10.1515/bot-20120150.

[18] Rathore, S. S., Chaudhary, D. R., Boricha, G. N., Ghosh, A., Bhatt, B. P., Zodape, S. T., Patolia, J. S. (2009): Effect of seaweed extract on the growth, yield and nutrient uptake of soybean (Glycine max) under rainfed conditions. - South African Journal of Botany 75: 351-355.

[19] Rengasamy, K. R. R., Kulkarni, M. G., Stirk, W. A., Van Staden, J. (2015): Eckol - a new plant growth stimulant from the brown seaweed Ecklonia maxima. - Journal of Applied Phycology 27: 581-587. doi.:10.1007/s10811-014-0337-z.

[20] Rouphael, Y., De Micco, V., Arena, C., Raimondi, G., Colla, G., De Pascale, S. (2017): Effect of Ecklonia maxima seaweed extract on yield, mineral composition, gas exchange and leaf anatomy of zucchini squash grown under saline conditions. - Journal of Applied Phycology 29: 459-470.

[21] Sharma, H. S. S., Fleming, C., Selby, C., Rao, J. R., Martin, T. (2014): Plant biostimulants: a review on the processing of macroalgae and use of extracts for crop management to reduce abiotic and biotic stresses. - Journal of Applied Phycology 26: 465-490. doi.:10.1007/s10811-013-0101-9. 
[22] Sosnowski, J., Malinowska, E., Wiśniewska-Kadżajan, B., Jankowski, K. (2016): Evaluation of the impact of the Ecklonia maxima extract on selected morphological features of yellow pine, spruce and thuja stabbing. - Journal of Ecolological Engineering 17(3): 169-174. doi.:10.12911/22998993/63475.

[23] Sridhar, S., Rengasamy, R. (2011): Influence of seaweed liquid fertilizer on growth and biochemical characteristics of Arachis hypogea L. under field trial. - Journal of Ecobiotechnology 3(12): 18-22.

[24] Staugaitis, G., Rutkauskiene, R. (2010): Comparison of magnesium determination methods as influenced by soil properties. - Žemdirbystè=Agriculture 3(97): 105-116.

[25] Stirk, W. A., Aurthur, G. D., Lourens, A. F., Novak, O., Strnad, M., Van Staden, J. (2004): Changes in cytokinins and auxin concentrations in seaweed concentrates when stored at an elevated temperature. - Journal of Applied Phycology 16: 31-39. doi.:10.1023/B:JAPH.0000019057.45363.f5.

[26] Sultana, V., Ehteshamul-Haque, S., Ara, J., Athar, M. (2005): Comparative efficacy of Brown, Green and red Seaweeds in the control of Root infesting fungi and okra. International Journal of Environental Science and Technology 2(2): 129-132. doi.:10.1007/BF03325866.

[27] Szkutnik, J., Kacorzyk, P., Szewczyk, W. (2012): The content change of total protein and crude fibre depending on the dose of fertilization and phonological phase of grasses. Grassland Science in Poland 15: 185-191. (In Polish, abstract in English).

[28] Virkajärvi, P., Saarijärvi, K., Rinne, M., Saastamoinen, M. (2012): Grass physiology and its relations to nutritive value in feeding horses. - In: Saastamoinen, M., Fradinho, M. J., Santos, A. S., Miraglia, N. (eds.) Forages and grazing in horse nutrition, vol. 132. Wageningen Academic Publishers. 